\title{
Visible T1-hyperintensity of the dentate nucleus after multiple administrations of macrocyclic gadolinium-based contrast agents: yes or no?
}

Alessandra Splendiani", Antonella Corridore, Silvia Torlone, Milvia Martino, Antonio Barile, Ernesto Di Cesare and Carlo Masciocchi

\begin{abstract}
Objectives: To investigate the appearance of visible dentate nucleus (DN) T1-hyperintensity and quantify changes in DN/pons (DN/P) signal intensity (SI) ratio in MS patients after the exclusive administration of macrocyclic GBCAs.

Materials and methods: One hundred forty-nine patients with confirmed MS were evaluated. Patients received at least two administrations of gadobutrol $(n=63)$, gadoterate $(n=57)$, or both $(n=29)$. Two experienced neuroradiologists in consensus evaluated unenhanced T1-weighted MR images from all examinations in each patient for evidence of visible DN hyperintensity. Thereafter, SI measurements were made in the left and right DN and pons on unenhanced T1-weighted images from the first and last scans. A two-sample $t$ test compared the DN/P SI ratios for patients with and without visible T1-hyperintensity.

Results: Visible T1-hyperintensity was observed in 42/149 (28.2\%) patients (19 after gadobutrol only, 15 after gadoterate only, 8 after both), typically at the 4th or 5th follow-up exam at 3-4 years after the initial examination. Significant increases in DN/P SI ratio from first to last examination were determined for patients with visible T1-hyperintensity $(0.998 \pm 0.002$ to $1.153 \pm 0.016, p<0.0001$ for gadobutrol; $1.003 \pm 0.004$ to $1.110 \pm 0.014, p<0.0001$ for gadoterate; $1.004 \pm 0.011$ to $1.163 \pm 0.032, p=0.0004$ for both) but not for patients without visible T1-hyperintensity ( $p>0.05$; all groups).
\end{abstract}

Conclusion: Multiple injections of gadobutrol and/or gadoterate can lead to visible and quantifiable increases in DN/P SI ratio in some patients with MS.

Keywords: Gadolinium-based contrast agents, Gadolinium retention, Dentate nucleus, Gadoterate dimeglumine, Gadobutrol

\section{Key points}

- Visible T1-hyperintensity of the dentate nucleus on unenhanced T1-weighted images of MS patients can occur after the exclusive administration of the macrocyclic GBCAs Dotarem and Gadovist.

- Visible T1-hyperintensity is associated with significant increases in DN/P SI ratio.

\footnotetext{
* Correspondence: alessandra.splendiani@cc.univaq.it

Department of Biotechnological and Applied Clinical Sciences, University of L'Aquila, 67100 L'Aquila, Italy
}

- 11Variation of changes in DN/P SI ration across patients suggests the appearance of visible T1hyperintensity is a patient-specific process rather than a GBCA-specific process.

- There is no evidence that the appearance of visible T1-hyperintensity or changes in DN/P SI ratio has any impact on patient health or safety.

\section{Introduction}

High signal intensity (SI) in the dentate nucleus (DN) and globus pallidus (GP) on unenhanced T1-weighted MR images following the cumulative administration of exclusively 
macrocyclic gadolinium-based contrast agents (GBCAs) is currently a source of debate and controversy. The widely held view is that visible T1-hyperintensity occurs primarily after the cumulative administration of so-called linear GBCAs rather than macrocyclic GBCAs and that the observed high T1-signal possibly reflects the release of gadolinium $(\mathrm{Gd})$ from the linear chelating molecule and its subsequent retention in the brain, possibly through binding to cellular macromolecules [1-6]. Although no clinical signs or symptoms attributable to brain Gd retention have yet been observed after cumulative exposure to any GBCA, linear or macrocyclic [7-11], the widespread assumption is that retained Gd presumed to have been released from linear GBCAs and "visible" on unenhanced T1-weighted images potentially poses a long-term risk to human health [12] while the absence of manifest T1-signal increases with macrocyclic GBCAs is taken as indicating their inherent "safety". A consequence of this assumption in Europe has been the suspension or restriction of certain linear GBCAs for clinical use [12].

In recent years, numerous studies have demonstrated T1-signal increases in the brain after the exclusive administration of macrocyclic GBCAs [13-19]. Invariably, however, these studies have been criticized and marginalized, often by the manufacturers of the GBCA in question [20-23], in part, because the images presented do not show obvious T1-hyperintensity of the DN or GP to the extent seen with linear GBCAs [20]. Unfortunately, such criticism overlooks the fact that brain $\mathrm{Gd}$ retention has unequivocally been demonstrated not only in myriad animal studies [24-31] but also in human brain autopsy samples after the exclusive administration of macrocyclic GBCAs [32].

Patients with relapsing-remitting multiple sclerosis (RR-MS) typically undergo frequent GBCA-enhanced MR studies as part of their routine clinical follow-up. Studies looking at T1-signal changes in the DN and/or GP of patients with RR-MS after the exclusive serial administration of macrocyclic GBCAs have yielded conflicting results. Whereas some studies have failed to observe T1-signal changes [33-38], others have noted marked quantitative changes $[13,17]$. Recently, Splendiani et al. [15] reported visible T1-hyperintensity in the DN in roughly one third of patients with RR-MS who had received cumulative doses of the macrocyclic GBCAs gadobutrol and/or gadoterate. The aim of our study was to further investigate patients with RR-MS for the presence of visible signal enhancement of the DN on unenhanced T1-weighted images following multiple exposures to macrocyclic GBCAs.

\section{Methods and materials}

\section{Patients}

This single-center retrospective study was approved by the institutional review board and the need for patient informed consent was waived. We reviewed our PACS database for all patients with confirmed RR-MS who underwent two or more MRI exams exclusively at our center with the macrocyclic GBCAs gadoterate meglumine (Dotarem; Guerbet, Aulnay-sous-Bois, France) and/or gadobutrol (Gadovist; Bayer Healthcare, Berlin, Germany) between January 2005 and December 2017. Patients were included only if complete documentation regarding contrast administration (i.e., date of exam, type, and dose of GBCA administered) was available for each MRI exam. Patients were ineligible for inclusion if they had an eGFR of $\leq 60 \mathrm{~mL} / \mathrm{min} / 1.73 \mathrm{~m}^{2}$ based on a blood sample taken close to the date of the last MR examination; a history of cerebrovascular events including hemorrhage, stroke, or brain ischemia; previous cerebral neurosurgical treatments or radiation therapy; intra-axial brain tumor or other lesions such as vascular malformations located in the cerebellum or pons; a history of intracranial infection, such as meningitis or encephalitis; abnormal liver function or hepatic deficiency. Abnormal liver function was defined as the alteration of one or more of serum parameters as alanine aminotransferase, $\gamma$-glutamyl transpeptidase, or aspartate aminotransferase. Hepatic deficiency was considered an exclusion criterion as it can modify GBCA pharmacokinetics and because high signal intensity in basal ganglia on T1-weighted images is associated with hepatic cirrhosis and other forms of liver disease [39, 40].

For all included patients the age, sex, number of GBCA administrations, and accumulated dose were recorded. An estimation of the mean interval ( \pm standard deviation) between GBCA administrations was calculated by dividing the time in weeks between the first and last MRI examinations by the total number of GBCA administrations made during that period.

\section{MRI protocol}

All MRI exams were performed on a $3 \mathrm{~T}$ scanner (GE Healthcare-Signa EXCITE). Axial T1-weighted spinecho images were acquired before and after GBCA injection with repetition time $(\mathrm{TR})=500-588 \mathrm{~ms}$, echo time $(\mathrm{TE})=7.7-12 \mathrm{~ms}$, matrix $=256 \times 256$, section thickness $=4 \mathrm{~mm}$, and field of view $(\mathrm{FOV})=250$ $\mathrm{mm}$. Axial T2-weighted images were acquired with $\mathrm{TR}=4890 \mathrm{~ms}, \mathrm{TE}=85 \mathrm{~ms}$, section thickness $=4 \mathrm{~mm}$, matrix $=256 \times 256$ and FOV $=250 \mathrm{~mm}$. Axial susceptibility weighted images (SWI) were acquired with TR/ $\mathrm{TE}=85.2 / 32.6 \mathrm{~ms}$, a slab of 64 slices of $3 \mathrm{~mm}$ thickness, no gap, FOV $=220 \mathrm{~mm}$, an acquisition matrix of $384 \times 256$, number of excitations $(\mathrm{NEX})=2$, and a flip angle of $20^{\circ}$. Fluid-attenuated inversion recovery (FLAIR) and T1-weighted non-contrast sequences were used to precisely identify the $\mathrm{DN}$ and 
verify visible T1-hyperintensity after multiple contrast administrations.

All patients received a standardized dose of $0.1 \mathrm{mmol} / \mathrm{kg}$ body weight of either gadoterate or gadobutrol for each examination.

\section{Data collection}

Two neuroradiologists in consensus (AS and AC with 25 and 4 years' experience, respectively) evaluated unenhanced T1-weighted images from each MRI examination in each patient for visible T1-hyperintensity in the DN. Patients without visible T1-hyperintensity on any image were considered negative whereas patients with clearly discernable T1-hyperintensity of the $\mathrm{DN}$ relative to the pons were considered positive. Full details of the visible T1-hyperintensity, including the examination in which visible T1-hyperintensity first appeared and the total GBCA dose and time interval between examinations, was recorded for each positive patient.

Thereafter, SI values were determined in operatordefined oval regions-of-interest (ROI) positioned within the DN and pons of all positive patients, as described by Kanda et al. [1]. ROIs were made as large as possible (mean size, $10 \mathrm{~mm}^{2}$; range, 6-18 $\mathrm{mm}^{2}$ ) on both the left and right DN with care taken to avoid pulsating vessels (if present) and rim aspects. ROIs in the central pons were adjusted as appropriate to ensure homogeneity. The left and right DN visible on T2-FLAIR images (and, if required, the SWI images) were used to guide accurate ROI positioning on the corresponding unenhanced T1-weighted images.

\section{Statistical analysis}

Unpaired $t$ tests were performed to compare the mean DN/Pons (DN/P) SI ratio on unenhanced T1-weighted images at the last MRI examination with the DN/P SI ratio prior to the first contrast-enhanced examination. Separate analyses were performed for patients considered positive for visible T1-hyperintensity and for patients considered negative for visible T1-hyperintensity. The DN/P SI ratio for each patient was calculated after averaging the SI values in the left and right DN. Within each group, separate analyses were performed for all patients combined, for patients that received only gadoterate, for patients that received only gadobutrol, and for patients that received both gadoterate and gadobutrol during imaging follow-up. A significant change in DN/P SI ratio was considered for $p<0.05$.

\section{Results}

A summary of results is presented in Table 1. A total of 149 patients with confirmed RR-MS underwent two or more MRI exams exclusively at our center with gadoterate meglumine $(n=57)$, gadobutrol $(n=63)$, or both $(n=29)$. These 149 patients included 107 (71.8\%) for which visible T1-hyperintensity was not evident on any follow-up MRI exam and $42(28.2 \%)$ for which visible T1-hyperintensity was noted. Highly significant $(p \leq 0.0004)$ quantitative differences in mean DN/P SI ratio were noted for all groups in which visible T1-hyperintensity was noted. Conversely, no significant differences in mean DN/P SI ratio were apparent in any group in which visible T1-hyperintensity was not observed.

The number of administrations per patient in each subgroup is presented in Table 2. A tendency towards a lower number of GBCA administrations per patient was noted

Table 1 Summary of patients with and without visible T1-hyperintensity of the DN and changes in DN/P SI ratio

\begin{tabular}{|c|c|c|c|c|c|c|c|c|c|c|}
\hline Patients & $\begin{array}{l}\text { Patient } \\
\text { sub-group }\end{array}$ & $\begin{array}{l}\text { Mean } \\
\text { age } \pm S D\end{array}$ & $\begin{array}{l}M / \\
F\end{array}$ & $\begin{array}{l}\text { No. of } \\
\text { exams }\end{array}$ & $\begin{array}{l}\text { Exam no. at } \\
\text { first } \\
\text { appearance }\end{array}$ & $\begin{array}{l}\text { Mean interval } \\
\text { between exams } \\
\text { (months) }\end{array}$ & $\begin{array}{l}\text { Time to first } \\
\text { appearance } \\
\text { (months) }\end{array}$ & $\begin{array}{l}\text { Mean DN/P } \\
\text { before first } \\
\text { exam }\end{array}$ & $\begin{array}{l}\text { Mean DN/P } \\
\text { before last } \\
\text { exam }\end{array}$ & $\begin{array}{l}\text { Unpaired } \\
t \text { test }\end{array}$ \\
\hline \multirow[t]{4}{*}{ Negative } & All & $51 \pm 10.5$ & $\begin{array}{l}26 / \\
81\end{array}$ & $6.75 \pm 3.43$ & $\mathrm{n} / \mathrm{a}$ & $10.6 \pm 6.2$ & $\mathrm{n} / \mathrm{a}$ & $1.025 \pm 0.006$ & $1.032 \pm 0.005$ & $p=0.323$ \\
\hline & Gadobutrol & $54 \pm 8.5$ & $\begin{array}{l}12 / \\
32\end{array}$ & $7.34 \pm 3.82$ & $\mathrm{n} / \mathrm{a}$ & $10.43 \pm 8.3$ & $\mathrm{n} / \mathrm{a}$ & $1.045 \pm 0.008$ & $1.046 \pm 0.008$ & $p=0.970$ \\
\hline & Gadoterate & $47 \pm 11.5$ & $\begin{array}{l}8 / \\
34\end{array}$ & $7.21 \pm 2.86$ & $\mathrm{n} / \mathrm{a}$ & $11.3 \pm 4.5$ & $\mathrm{n} / \mathrm{a}$ & $1.001 \pm 0.004$ & $1.005 \pm 0.003$ & $\begin{array}{l}p= \\
0.4239\end{array}$ \\
\hline & Both & $49 \pm 6.3$ & $\begin{array}{l}6 / \\
15\end{array}$ & $4.62 \pm 4.6$ & $\mathrm{n} / \mathrm{a}$ & $9.8 \pm 6.7$ & $\mathrm{n} / \mathrm{a}$ & $1.027 \pm 0.025$ & $1.06 \pm 0.014$ & $p=0.543$ \\
\hline \multirow[t]{4}{*}{ Positive } & All & $50 \pm 11.5$ & $\begin{array}{l}8 / \\
42\end{array}$ & $10.21 \pm 4.6$ & $4.36 \pm 2.5$ & $10.79 \pm 6.92$ & $43.48 \pm 27.34$ & $1.003 \pm 0.004$ & $1.137 \pm 0.012$ & $\begin{array}{l}p< \\
0.0001\end{array}$ \\
\hline & Gadobutrol & $51 \pm 11.2$ & $\begin{array}{l}1 / \\
19\end{array}$ & $11.16 \pm 5.4$ & $4.79 \pm 2.5$ & $10.9 \pm 6.4$ & $48.32 \pm 28.7$ & $0.998 \pm 0.002$ & $1.153 \pm 0.016$ & $\begin{array}{l}p< \\
0.0001\end{array}$ \\
\hline & Gadoterate & $42 \pm 8.4$ & $\begin{array}{l}6 / \\
15\end{array}$ & $9.07 \pm 3.9$ & $3.93 \pm 2.4$ & $11.0 \pm 8.0$ & $40.0 \pm 27.32$ & $1.003 \pm 0.004$ & $1.110 \pm 0.014$ & $\begin{array}{l}p< \\
0.0001\end{array}$ \\
\hline & Both & $60 \pm 6.4$ & $1 / 8$ & $10.13 \pm 4.7$ & $4.13 \pm 2.8$ & $10.2 \pm 6.7$ & $38.5 \pm 25.5$ & $1.004 \pm 0.011$ & $1.163 \pm 0.032$ & $\begin{array}{l}p= \\
0.0004\end{array}$ \\
\hline
\end{tabular}


Table 2 Number of GBCA administrations given to patients with and without visible T1-hyperintensity of the DN

\begin{tabular}{|c|c|c|c|c|c|c|c|c|c|c|c|c|c|c|c|c|c|c|c|c|c|c|c|}
\hline \multirow[t]{2}{*}{ Group } & \multirow[t]{2}{*}{ Sub-group } & \multicolumn{21}{|c|}{ Number of administrations } & \multirow{2}{*}{$\begin{array}{l}\text { No. of } \\
\text { patients }\end{array}$} \\
\hline & & 2 & 3 & 4 & 5 & 6 & 7 & 8 & 9 & 10 & 11 & 12 & 13 & 14 & 15 & 16 & 17 & 18 & 19 & 20 & 21 & 22 & \\
\hline \multirow[t]{4}{*}{ Negative } & All & 10 & 7 & 14 & 12 & 11 & 13 & 13 & 8 & 7 & 3 & 3 & - & 1 & 4 & - & - & - & - & 1 & - & - & 107 \\
\hline & Gadobutrol & 3 & 2 & 6 & 4 & 5 & 6 & 5 & 3 & 4 & - & 2 & - & 1 & 2 & - & - & - & - & 1 & - & - & 44 \\
\hline & Gadoterate & 1 & 1 & 5 & 6 & 4 & 7 & 8 & 3 & 2 & 2 & 1 & - & - & 2 & - & - & - & - & - & - & - & 42 \\
\hline & Both & 6 & 4 & 3 & 2 & 2 & - & - & 2 & 1 & 1 & - & - & - & - & - & - & - & - & - & - & - & 21 \\
\hline \multirow[t]{4}{*}{ Positive } & All & - & 2 & 2 & 3 & 3 & 1 & 4 & 4 & 4 & 6 & 3 & 2 & 3 & 1 & - & - & 1 & - & - & 2 & 1 & 42 \\
\hline & Gadobutrol & - & 1 & - & 1 & 2 & - & 2 & 2 & 2 & 3 & - & 1 & 2 & - & - & - & - & - & - & 2 & 1 & 19 \\
\hline & Gadoterate & - & 1 & 1 & 1 & 1 & 1 & 1 & 1 & 1 & 3 & 3 & - & - & 1 & - & - & - & - & - & - & - & 15 \\
\hline & Both & - & - & 1 & 1 & - & - & 1 & 1 & 1 & - & - & 1 & 1 & - & - & - & 1 & - & - & - & - & 8 \\
\hline
\end{tabular}

for patients with no evidence of visible T1-hyperintensity: 43/107 (40.2\%) patients without evidence of visible T1hyperintensity received five or fewer GBCA administrations compared with only $7 / 42$ (16.7\%) patients with evidence of visible T1-hyperintensity. Typically, the first appearance of visible T1-hyperintensity in positive patients occurred at the fourth or fifth follow-up exam, between approximately 3 and 4 years after the initial MRI examination. Few differences were apparent between groups in terms of the time to appearance of the first visible hyperintensity.

Examples of the T1-hyperintensity seen after the exclusive administration of gadoterate meglumine and gadobutrol are shown in Figs. 1 and 2, respectively. The increase in DN/P SI ratio from first to last MRI exam in patients with visible T1-hyperintensity is shown in Figs. 3 and 4, respectively.

\section{Discussion}

Although numerous studies have reported quantitative changes in T1-signal in the $\mathrm{DN}$ and/or GP following cumulative administration of macrocyclic GBCAs [13-19, 41, 42], comparatively few $[15,16,18,42]$ have reported visible T1-hyperintensity. Those studies that have reported visible T1-signal changes following the administration of macrocyclic GBCAs have largely been overlooked or disregarded, even in official guidelines and recommendations [12]. The results of our study provide clear evidence that T1-signal changes can and do occur in the DN after multiple administrations of the macrocyclic GBCAs, gadobutrol and gadoterate meglumine, and that the appearance of visible T1-hyperintensity is associated with significant increases in DN/P SI ratio.

To note is that not all patients that received multiple administrations of macrocyclic GBCAs demonstrated visible T1-hyperintensity. In common with the findings of many previous studies [3-6], 107/149 (71.8\%) patients showed no evidence of visible hyperintensity, even after as many as 20 GBCA administrations. However, it should be noted that patients that demonstrated no signs of visible T1-hyperintensity tended to have received fewer GBCA administrations overall. The appearance of visible T1-hyperintensity in our cohort tended to occur at the 4th or 5th MRI examination whereas approximately $40 \%$ of patients negative for visible T1-hyperintensity received five or fewer MRI examinations. It is unclear why some patients demonstrated clear visible T1-hyperintensity of the DN while others did not. Likewise, it is unclear why the relative change in DN/P SI ratio from first to last GBCA administration differed across patients in terms of both magnitude and time course or why the appearance of visible T1-hyperintensity in the DN showed no obvious correlation with quantitative DN/P SI ratio. A recent study by Moreno et al. [18] in melanoma patients referred for serial follow-up gadobutrol-enhanced MRI similarly revealed visible T1hyperintensity in some, but not all, patients without any association between the $\mathrm{DN} / \mathrm{P}$ SI ratio and the dose administered or the number of MR examinations. In common with our study, Moreno et al. [18] found a significantly $(p=0.024)$ higher $\mathrm{DN} / \mathrm{P}$ SI ratio in patients with visible DN T1-hyperintensity than in patients without visible T1-hyperintensity. Other authors have similarly revealed differences over time in terms of DN/P SI ratio with marked differences across patients in terms of the magnitude of changes [43].

Importantly, none of the patients in our cohort had hepatic deficiency or other clinical issues which might explain a differential appearance of DN T1-hyperintensity and none had had previous cerebral neurosurgical treatments or radiation therapy which might explain the appearance of T1-hyperintensity [39, 40, 44-46]. Furthermore, there were no relevant differences between SI measurements in the left and right DN of each patient that might suggest a direct association with MS disease progression. On the other hand, MS is a progressive neurodegenerative disease for which patients undergo frequent follow-up MRI examinations and for which disease progression is a potential confounding variable [8]. An early study reported more 


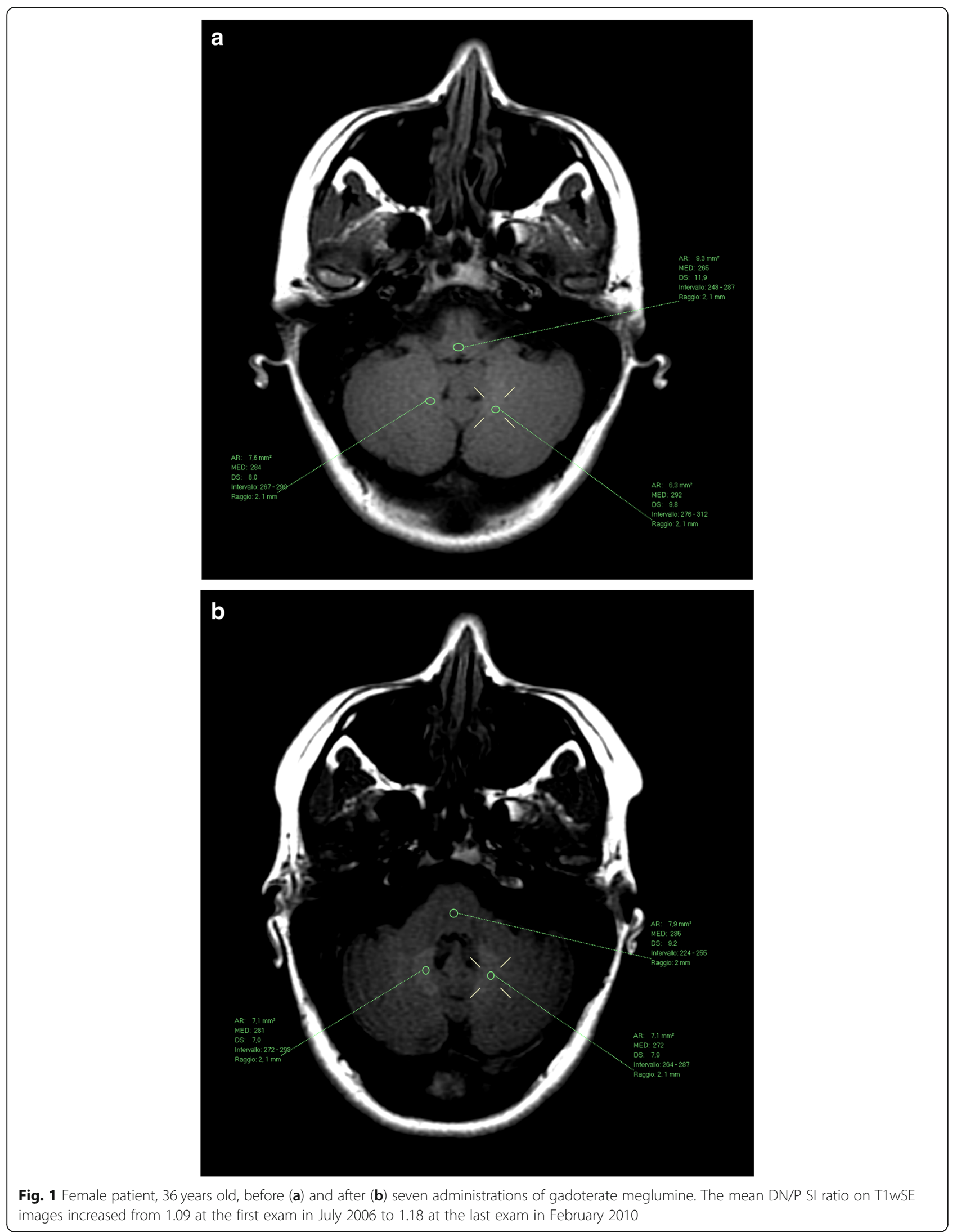




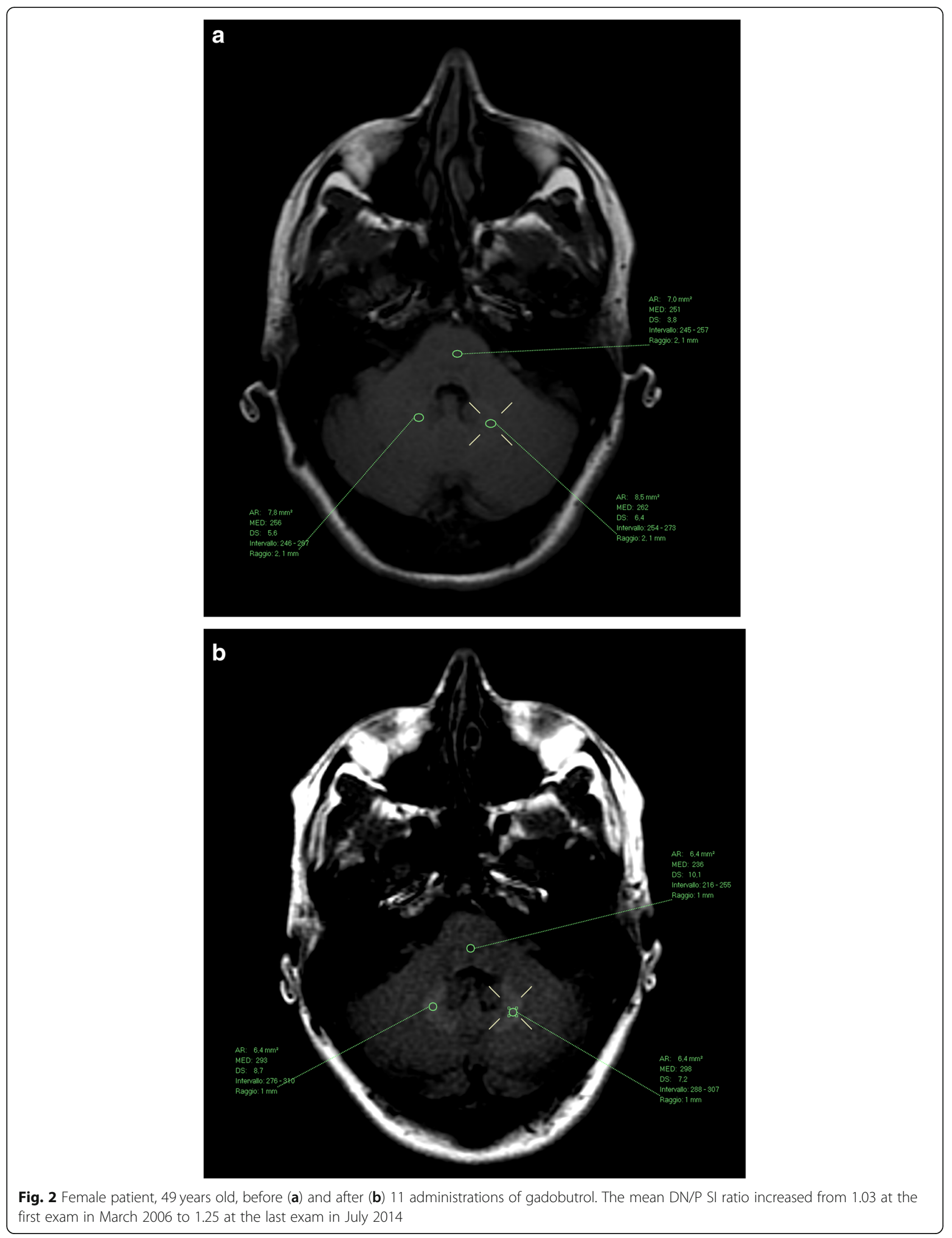




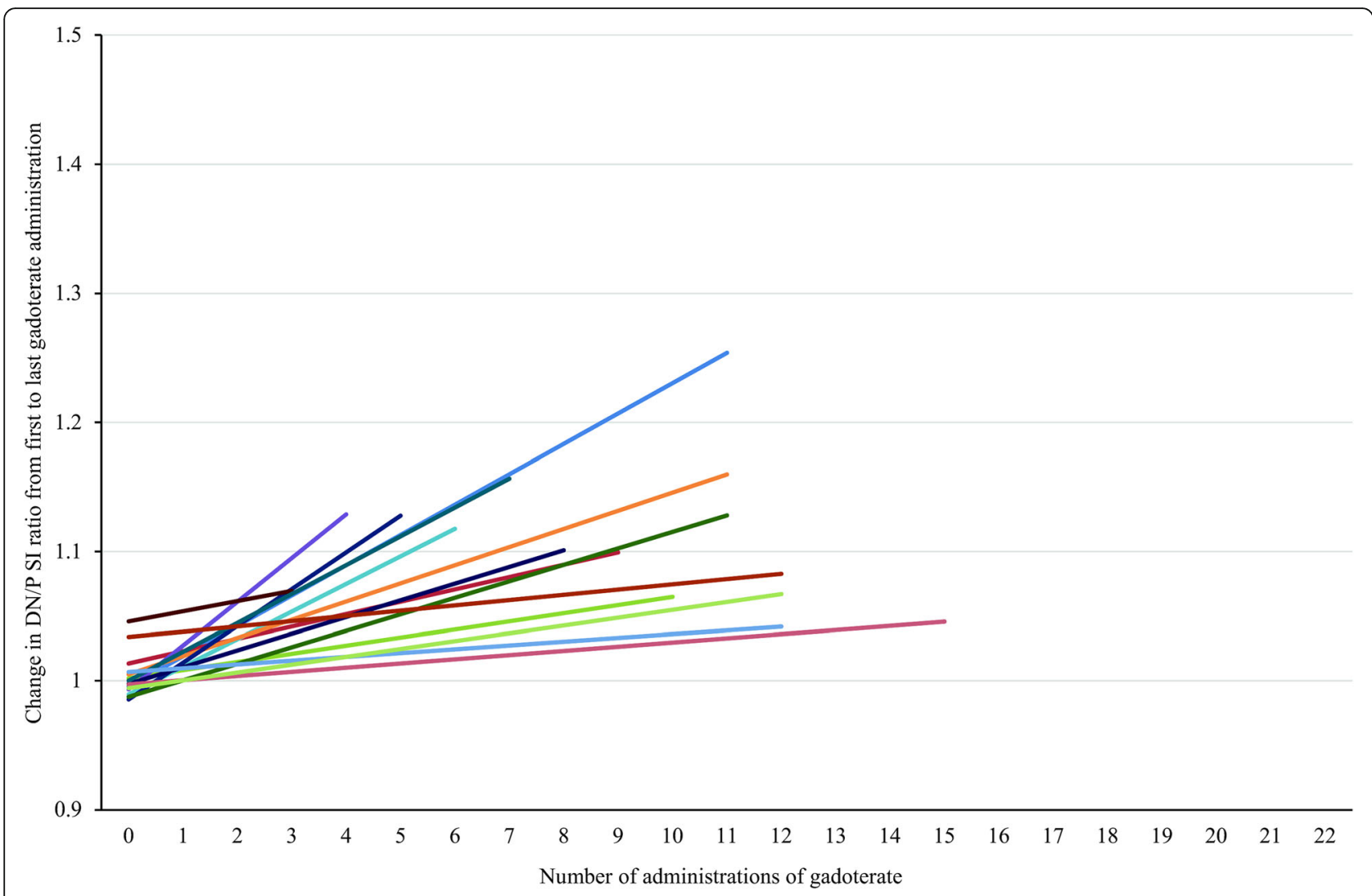

Fig. 3 First and last DN/P SI ratios for patients with visible T1-signal changes after two or more administrations of gadoterate meglumine

frequent T1-hyperintensity of the DN in patients with secondary-progressive MS than in patients with primary progressive disease (46\% versus $8 \%$, respectively) and found that this correlated with a higher score on the Expanded Disability Status Scale (EDSS), a higher brain lesion load, and greater tissue loss [47]. However, whereas contrastenhanced T1-weighted images were acquired with either gadoterate or gadodiamide (Omniscan, GE Healthcare) no information was provided on prior GBCA administrations, so it is difficult to determine whether the observed T1-hyperintensity reflected differences in the stage/extent of disease or differences in the amount/type of GBCA administered. Although some studies [48, 49] have looked to correlate T1-hyperintensity of the DN in patients with MS with loss of verbal fluency and cognition, it is difficult to separate a hypothetic effect of Gd retention from the normal effects of MS progression, especially given the difficulties in finding a matched MS group not exposed to any GBCA. Recent studies by Cocozza et al. [9] and Ackermans et al. [10] suggest that MRI features suggestive of Gd retention do not correlate with EDSS worsening in patients with MS. These findings are supported by studies that have shown no effects of DN T1-hyperintensity on DN tissue integrity $[11,50,51]$.
Our findings support those of Stojanov et al. [13] and Splendiani et al. [15] in showing significant increases in DN/P SI ratio following the cumulative administration of macrocyclic GBCAs in patients with RR-MS. Conversely, they are at variance with the findings of others [33-38]. The clear disparity in study findings between research groups highlights the complexity of the issues at hand and the need for carefully controlled large-scale studies to investigate the processes underlying T1-signal changes in these patients. Studies are needed to ascertain whether T1-hyperintensity in the DN is in fact indicative of $\mathrm{Gd}$ retention or whether it represents some other process specific to MS and/or other diseases. In the absence of histologic verification of Gd retention, no conclusions can be made as to the processes underlying the T1-signal changes observed in our patient cohort. However, this is the same for all studies that focus solely on observations from imaging studies.

The strength of our study lies in the fact that all patients received only macrocyclic GBCAs and that imaging was performed in a carefully monitored manner exclusively at our center. Therefore, the possibility that patients with 


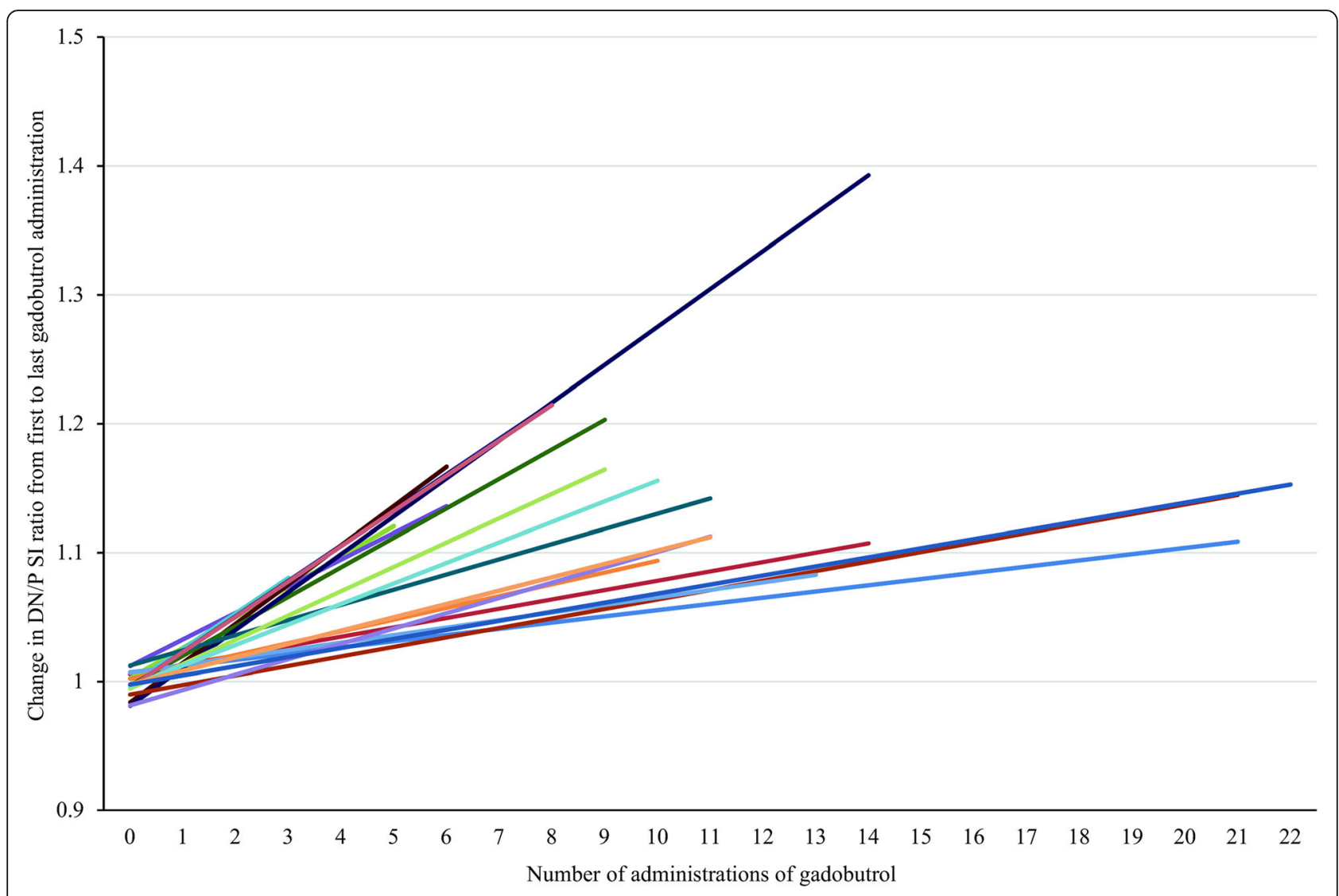

Fig. 4 First and last DN/P SI ratios for patients with visible T1-signal changes after two or more administrations of gadobutrol

visible T1-hyperintensity could have received prior administrations of certain linear GBCAs that are more widely associated with $\mathrm{T} 1$-signal changes in the $\mathrm{DN}$ can be excluded. Secondly, images from all patients that received at least two macrocyclic GBCA administrations were carefully assessed for visible T1-hyperintensity by two neuroradiologists in consensus. Prior studies to evaluate T1-signal changes have frequently limited the number of patients to a convenient round number (e.g., $n=50$ [6]), restricted patient inclusion to only those that received a minimum number of GBCA administrations (e.g., $n=6$ ), and cited SI values determined by just a solitary reader. Our approach avoided any form of selection bias and was based solely on the visual appearance of the DN relative to the pons. Our approach therefore more typically reflects the assessment process routinely undertaken in clinical practice. Finally, our study included patients with RR-MS evaluated exclusively at our center over an extended period (approximately 13 years). Other studies have drawn conclusions based on very few evaluated patients imaged over a relatively short time frame [37].

A limitation of our study is the absence of an agematched control group of MS patients that had never been exposed to any GBCA. However, such patients would be extremely difficult to find given that contrast-enhanced MRI is one of the principal imaging techniques for MS patients, both for initial diagnosis and follow-up. A second limitation is that we did not quantify the DN/P SI ratio at every follow-up exam and thus were unable to detail a time course for T1-signal changes in individual patients. However, in quantifying changes in DN/P SI ratio from SI measurements determined prior to the first and last contrast-enhanced exam in each patient, we showed that increases in DN/P SI ratio can and do occur after both gadoterate and gadobutrol and that the changes in T1-signal are variable across patients both in terms of magnitude and time course. Unfortunately, a statistical comparison of these two GBCAs in terms of the changes observed was beyond the scope of this initial study given the relatively small number of positive patients in each group. Finally, we did not attempt to correlate the appearance of visible T1-hyperintensity with cognitive or physical disability as performed elsewhere $[9,10,49]$. Moreover, we did not look at a possible correlation with brain lesion load and tissue loss. This was considered beyond the scope of this initial investigation but should be considered for further large-scale studies in MS patients. Further 
research should additionally look at changes in R1 relaxation rates which are less prone to potential sources of inaccuracy.

In conclusion, we observed visible T1-hyperintensity of the DN in 42/149 (28.2\%) patients with RR-MS who had received at least two administrations of gadobutrol, gadoterate, or both. The appearance of visible T1-hyperintensity correlated with significant increases in DN/P SI ratio from first to last examination.

\section{Abbreviations}

DN: Dentate nucleus; eGFR: Estimated glomerular filtration rate; FLAIR: Fluid-attenuated inversion recovery; FOV: Field of view; GBCA: Gadolinium-based contrast agent; GP: Globus pallidus; MS: Multiple sclerosis; NEX: Number of excitations; P: Pons; ROI: Region of interest; SI: Signal intensity; SWI: Susceptibility weighted images; TE: Echo time; TR: Repetition time

\section{Acknowledgements}

Not applicable

\section{Authors' contributions}

$A S$ is the principal investigator on the study. $A C, S T, M M$, and $A B$ were responsible for the acquisition of images. $A S$ and $A C$ evaluated patient images and performed the analyses. EDC and CM reviewed performed data review and manuscript revision. All authors read and approved the final manuscript.

\section{Funding}

This research did not receive any specific grant from funding sources in the public, commercial, or not-for-profit sectors.

\section{Availability of data and materials}

All data on patients with confirmed RR-MS are taken from our PACS database.

\section{Ethics approval and consent to participate}

Our study was approved by the local Ethics committee of our center.

Requirement for patient approved consent was waived.

\section{Consent for publication}

Not applicable

\section{Competing interests}

The authors declare that they have no competing interests.

Received: 17 May 2019 Accepted: 11 July 2019

Published online: 04 September 2019

\section{References}

1. Kanda T, Ishii K, Kawaguchi H, Kitajima K, Takenaka D (2014) High signal intensity in the dentate nucleus and globus pallidus on unenhanced T1-weighted MR images: relationship with increasing cumulative dose of a gadolinium-based contrast material. Radiology 270:834-841

2. Errante $Y$, Cirimele V, Mallio CA, Di Lazzaro V, Zobel BB, Quattrocchi CC (2014) Progressive increase of T1 signal intensity of the dentate nucleus on unenhanced magnetic resonance images is associated with cumulative doses of intravenously administered gadodiamide in patients with normal renal function, suggesting dechelation. Invest Radiol 49:685-690

3. Kanda T, Osawa M, Oba H et al (2015) High signal intensity in dentate nucleus on unenhanced T1-weighted MR images: association with linear versus macrocyclic gadolinium chelate administration. Radiology 275:803-809

4. Radbruch A, Weberling LD, Kieslich PJ et al (2016) Intraindividual analysis of signal intensity changes in the dentate nucleus after consecutive serial applications of linear and macrocyclic gadolinium-based contrast agents. Invest Radiol 51:683-690
5. Radbruch A, Weberling LD, Kieslich PJ et al (2015) Gadolinium retention in the dentate nucleus and globus pallidus is dependent on the class of contrast agent. Radiology 275:783-791

6. Guo BJ, Yang ZL, Zhang LJ (2018) Gadolinium deposition in brain: current scientific evidence and future perspectives. Front Mol Neurosci 11:335

7. Welk B, McArthur E, Morrow SA et al (2016) Association between gadolinium contrast exposure and the risk of parkinsonism. JAMA 316:96-98

8. Gulani V, Calamante F, Shellock FG et al (2017) Gadolinium deposition in the brain: summary of evidence and recommendations. Lancet Neurol 16:564-570

9. Cocozza S, Pontillo G, Lanzillo R et al (2019) MRI features suggestive of gadolinium retention do not correlate with expanded disability status scale worsening in multiple sclerosis. Neuroradiology 61:155-162

10. Ackermans N, Taylor C, Tam R et al (2019) Effect of different doses of gadolinium contrast agent on clinical outcomes in MS. Mult Scler J Exp Transl Clin. https://doi.org/10.1177/2055217318823796

11. Mallio CA, Piervincenzi C, Gianolio E et al (2019) Absence of dentate nucleus resting-state functional connectivity changes in nonneurological patients with gadolinium-related hyperintensity on T1-weighted images. J Magn Reson Imaging. https://doi.org/10.1002/jmri.26669

12. (2017) EMA's final opinion confirms restrictions on use of linear gadolinium agents in body scans. Available via https://www.ema.europa.eu/documents/ referral/gadolinium-article-31-referral-emas-final-opinion-confirmsrestrictions-use-linear-gadoliniumagents_en.pdf. Accessed 18 Apr 2019.

13. Stojanov DA, Aracki-Trenkic A, Vojinovic S, Benedeto-Stojanov D, Ljubisavljevic S (2016) Increasing signal intensity within the dentate nucleus and globus pallidus on unenhanced T1W magnetic resonance images in patients with relapsing-remitting multiple sclerosis: correlation with cumulative dose of a macrocyclic gadolinium-based contrast agent, gadobutrol. Eur Radiol 26:807-815

14. Rossi Espagnet MC, Bernardi B, Pasquini L, Figà-Talamanca L, Tomà $P$, Napolitano A (2017) Signal intensity at unenhanced T1-weighted magnetic resonance in the globus pallidus and dentate nucleus after serial administrations of a macrocyclic gadolinium-based contrast agent in children. Pediatr Radiol 47:1345-1352

15. Splendiani A, Perri M, Marsecano C et al (2018) Effects of serial macrocyclicbased contrast materials gadoterate meglumine and gadobutrol administrations on gadolinium-related dentate nuclei signal increases in unenhanced T1-weighted brain: a retrospective study in 158 multiple sclerosis (MS) patients. Radiol Med 123:125-134

16. Bjørnerud A, Vatnehol SAS, Larsson C, Due-Tønnessen P, Hol PK, Groote IR (2017) Signal enhancement of the dentate nucleus at unenhanced MR imaging after very high cumulative doses of the macrocyclic gadolinium-based contrast agent Gadobutrol: an observational study. Radiology 285:434-444

17. Kelemen P, Alaoui J, Sieron D et al (2018) T1-weighted grey matter signal intensity alterations after multiple administrations of gadobutrol in patients with multiple sclerosis, referenced to white matter. Sci Rep 8:16844

18. Moreno J, Vaz NB, Soler JC et al (2018) High signal intensity in the dentate nucleus on unenhanced T1-weighted MR images in melanoma patients receiving macrocyclic gadolinium-based contrast. J Radiol Diagn Methods 1 101-107

19. Gehweiler J, Re T, Amann M, Schadelin S, Cabezon R, Stieltjes B (2018) The gadolinium deposition debate revisited using a fully automated big data approach. Radiological Society of North America 2018 Scientific Assembly and Annual Meeting, 2018, Chicago. archive. rsna.org/2018/18015095.html. Accessed 18 Apr 2019

20. Agris J, Pietsch H, Balzer T (2016) What evidence is there that gadobutrol causes increasing signal intensity within the dentate nucleus and globus pallidus on unenhanced T1W MRI in patients with RRMS? Eur Radiol 26:816-817

21. Lancelot E, Raynaud JS, Desché P (2017) Lack of evidence of a relationship between magnetic resonance signal intensity changes in the globus pallidus and dentate nucleus, and repeated administrations of gadoterate meglumine in children. Pediatr Radiol 47:1692-1693

22. Raynaud JS, Darmon-Kern E, Lancelot E, Laurent AC, Desché $P$ (2018) Re: effects of serial macrocyclic-based contrast materials gadoterate meglumine and gadobutrol administrations on gadolinium-related dentate nuclei signal increases in unenhanced T1-weighted brain: a retrospective study in 158 multiple sclerosis (MS) patients. Radiol Med 123:432-433

23. Radbruch A, Quattrocchi CC (2017) Interpreting signal-intensity ratios without visible $T 1$ hyperintensities in clinical gadolinium retention studies. Pediatr Radiol 47:1688-1689 
24. Jost G, Lenhard DC, Sieber MA, Lohrke J, Frenzel T, Pietsch H (2016) Signal increase on unenhanced T1-weighted images in the rat brain after repeated, extended doses of gadolinium-based contrast agents: comparison of linear and macrocyclic agents. Invest Radiol 51:83-89

25. Robert P, Lehericy S, Grand S et al (2015) T1-weighted hypersignal in the deep cerebellar nuclei after repeated administrations of Gd-based contrast agents in healthy rats: difference between linear and macrocyclic agents. Invest Radiol 50:473-480

26. Frenzel T, Apte C, Jost G, Schöckel L, Lohrke J, Pietsch H (2017) Quantification and assessment of the chemical form of residual gadolinium in the brain after repeated administration of gadolinium-based contrast agents: comparative study in rats. Invest Radiol 52:396-404

27. Lohrke J, Frisk AL, Frenzel T et al (2017) Histology and gadolinium distribution in the rodent brain after the administration of cumulative high doses of linear and macrocyclic gadolinium-based contrast agents. Invest Radiol 52:324-323

28. Jost G, Frenzel T, Lohrke J, Lenhard DC, Naganawa S, Pietsch H (2017) Penetration and distribution of gadolinium-based contrast agents into the cerebrospinal fluid in healthy rats: a potential pathway of entry into the brain tissue. Eur Radiol 27:2877-2885

29. McDonald RJ, McDonald JS, Dai D et al (2017) Comparison of gadolinium concentrations within multiple rat organs after intravenous administration of linear versus macrocyclic gadolinium chelates. Radiology 285:536-545

30. Boyken J, Frenzel T, Lohrke J, Jost G, Pietsch H (2018) Gadolinium accumulation in the deep cerebellar nuclei and Globus pallidus after exposure to linear but not macrocyclic gadolinium-based contrast agents in a retrospective pig study with high similarity to clinical conditions. Invest Radiol 53:278-285

31. Bussi S, Coppo A, Botteron C et al (2018) Differences in gadolinium retention after repeated injections of macrocyclic MR contrast agents to rats. J Magn Reson Imaging 47:746-752

32. Murata N, Gonzalez-Cuyar LF, Murata K et al (2016) Macrocyclic and other non-group 1 gadolinium contrast agents deposit low levels of gadolinium in brain and bone tissue: preliminary results from 9 patients with normal renal function. Invest Radiol 51:447-453

33. Eisele P, Alonso A, Szabo K et al (2016) Lack of increased signal intensity in the dentate nucleus after repeated administration of a macrocyclic contrast agent in multiple sclerosis: an observational study. Medicine (Baltimore) 95(39):e4624

34. Schlemm L, Chien C, Bellmann-Strobl J et al (2017) Gadopentetate but not gadobutrol accumulates in the dentate nucleus of multiple sclerosis patients. Mult Scler 23:963-972

35. Eisele P, Szabo K, Alonso A et al (2018) Lack of T1 hyperintensity in the dentate nucleus after 15 administrations of a macrocyclic contrast agent in multiple sclerosis. J Neurol Neurosurg Psychiatry 89:324-326

36. Tedeschi E, Cocozza S, Borrelli P, Ugga L, Morra VB, Palma G (2018) Longitudinal assessment of dentate nuclei relaxometry during massive gadobutrol exposure. Magn Reson Med Sci 17:100-104

37. Jaulent P, Hannoun S, Kocevar G et al (2018) Weekly enhanced T1-weighted MRI with Gadobutrol injections in MS patients: is there a signal intensity increase in the dentate nucleus and the globus pallidus? Eur J Radiol 105:204-208

38. Hannoun S, Issa R, El Ayoubi NK et al (2018) Gadoterate meglumine administration in multiple sclerosis has no effect on the dentate nucleus and the globus pallidus signal intensities. Acad Radiol S1076-6332(18):30525-30527

39. Maeda H, Sato M, Yoshikawa A et al (1997) Brain MR imaging in patients with hepatic cirrhosis: relationship between high intensity signal in basal ganglia on T1-weighted images and elemental concentrations in brain. Neuroradiology 39:546-550

40. Mochizuki H, Kamakura K, Masaki T et al (1997) Atypical MRI features of Wilson's disease: high signal in globus pallidus on T1-weighted images. Neuroradiology 39:171-174

41. Kang KM, Choi SH, Hwang M, Yun TJ, Kim JH, Sohn CH (2018) T1 shortening in the globus pallidus after multiple administrations of gadobutrol: assessment with a multidynamic multiecho sequence. Radiology 287:258-266

42. Pasquini L, Rossi Espagnet MC, Napolitano A et al (2018) Dentate nucleus T1 hyperintensity: is it always gadolinium all that glitters. Radiol Med 123:469-473

43. Malhotra A, LeSar B, Wu X et al (2018) Progressive T1 shortening of the dentate nucleus in patients with multiple sclerosis: result of multiple administrations of linear gadolinium contrast agents versus intrinsic disease. AJR Am J Roentgenol 211:1099-1105

44. Prosch H, Grois N, Wnorowski M, Steiner M, Prayer D (2007) Long-term MR imaging course of neurodegenerative Langerhans cell histiocytosis. AJNR Am J Neuroradiol 28:1022-1028
45. Herynek V, Wagnerová D, Malucelli A, Vymazal J, Sameš M, Hájek M (2015) Alterations in the basal ganglia in patients with brain tumours may be due to excessive iron deposition. Oncol Lett 9:43-46

46. Kasahara S, Miki Y, Kanagaki M et al (2011) Hyperintense dentate nucleus on unenhanced T1-weighted MR images is associated with a history of brain irradiation. Radiology 258:222-228

47. Roccatagliata L, Vuolo L, Bonzano L, Pichiecchio A, Mancardi GL (2009) Multiple sclerosis: hyperintense dentate nucleus on unenhanced T1-weighted MR images is associated with the secondary progressive subtype. Radiology 251:503-510

48. Absinta M, Rocca MA, Filippi M (2011) Dentate nucleus T1 hyperintensity in multiple sclerosis. AJNR Am J Neuroradiol 32:E120-E121

49. Forslin Y, Shams S, Hashim F et al (2017) Retention of gadolinium-based contrast agents in multiple sclerosis: retrospective analysis of an 18-year longitudinal study. AJNR Am J Neuroradiol 38:1311-1316

50. Eisele P, Konstandin S, Szabo K et al (2017) Sodium MRI of T1 high signal intensity in the dentate nucleus due to gadolinium deposition in multiple sclerosis. J Neuroimaging 27:372-375

51. Eisele P, Szabo K, Ebert A et al (2019) Diffusion-weighted imaging of the dentate nucleus after repeated application of gadolinium-based contrast agents in multiple sclerosis. Magn Reson Imaging 58:1-5

\section{Publisher's Note}

Springer Nature remains neutral with regard to jurisdictional claims in published maps and institutional affiliations.

\section{Submit your manuscript to a SpringerOpen ${ }^{\circ}$ journal and benefit from:}

- Convenient online submission

- Rigorous peer review

- Open access: articles freely available online

High visibility within the field

- Retaining the copyright to your article

Submit your next manuscript at $\boldsymbol{\nabla}$ springeropen.com 